PAPER

\title{
Mirror movements in parkinsonism: evaluation of a new clinical sign
}

\section{A J Espay, J-Y Li, L Johnston, R Chen, A E Lang}

See Editorial Commentary, p 1330

J Neurol Neurosurg Psychiatry 2005;76:1355-1359. doi: 10.1136/jnnp.2005.062950

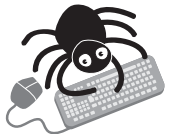

The supplemental data are available online at http//www.jnnp.com/ supplemental

Background: Mirror movements (MM) are not widely appreciated in parkinsonism and no report has evaluated this clinical sign in detail.

See end of article for authors' affiliations

Objectives: To define the parkinsonian clinical features associated with $M M$ in patients with early, asymmetric parkinsonism.

Correspondence to:

Dr A E Lang, Toronto

Western Hospital, Division

of Neurology, 399

Bathurst Street, MC 7-413,

Toronto, Ontario M5T

2S8, Canada; lang@

uhnres.utoronto.ca

Received 7 January 2005

Revised version received

5 April 2005

Accepted 12 April 2005

Methods: Twenty seven patients with early Parkinson's disease were evaluated using a standardised videotaping protocol. MM were scored from blinded video assessment using a clinical scale that rates the amplitude, distribution, and proportion of mirroring in the less affected limb. Parkinsonian features were combined into axial and lateralised scores using related items of the Unified Parkinson's Disease Rating Scale.

Results: MM were present in 24 of 27 patients. There was a significant linear correlation between the degree of asymmetry of motor deficits and MM on the less affected side. The effect of asymmetry was greater when the proportional rather than the absolute motor difference between sides was largest. Asymmetry in leg rigidity was the most important examination feature in the prediction of contralateral foot mirroring.

Conclusions: $M M$ are a clinical feature of the unaffected or less affected side in mild asymmetric parkinsonism. Their presence may be a useful clinical finding in early parkinsonism.

M irror movements (MM) are involuntary and unnecessary movements that accompany voluntary activity in homologous muscles on the opposite side of the body. They frequently involve the distal upper limbs during repetitive or alternating finger or hand movements. This phenomenon has been well recognised in normal early childhood and in a variety of hereditary and acquired disorders. ${ }^{1-8}$ Although no unifying theory accounting for its mechanism is entirely accepted, MM in such neurological disorders as X linked Kallmann's and childhood hemiparesis have been postulated to occur as a result of abnormal transcallosal inhibitory connections, relative overactivity of the ipsilateral motor cortex, failure of corticospinal tract decussation, or a combination of the above. ${ }^{9-12}$

MM have not been widely appreciated as a feature of parkinsonian disorders, although it is recognised that they may be present in corticobasal degeneration, typically in the more affected hand, when the clinically less affected hand is attempting to perform motor tasks. ${ }^{13}$ However, it is not generally documented that MM can be seen in the least affected limb in patients with typical Parkinson's disease (PD). We reported our preliminary findings on the association between MM and hemiparkinsonism in 2002. ${ }^{14}$ Subsequently, Vidal et al confirmed the presence of this phenomenon in the unaffected side of patients with mild hemiparkinsonism. ${ }^{15} \mathrm{We}$ now report our detailed clinical evaluation of this clinical sign in patients with early, asymmetric parkinsonism.

\section{MATERIALS AND METHODS Subjects}

Patients with suspected MM and recent onset, asymmetric parkinsonism consistent with idiopathic PD who were seen at the movement disorders centre, Toronto Western Hospital, Canada, between September 2001 and January 2003 were recruited. The university health network research ethics board approved our study and all patients signed informed consent. Exclusion criteria included evidence of associated lower motor neurone (atrophy, fasciculations, weakness, and hyporeflexia) or upper motor signs, previous neurosurgical procedures, severe resting tremor on the less affected side, which could preclude observation and scoring of mirroring, or clinical features that suggested a diagnosis other than PD including, but not restricted to, oculomotor disturbances, cognitive impairment, apraxia, and early falls. Although treatment with low doses of dopamine agonist monotherapy was permitted, patients treated with levodopa were generally excluded because of the potential for drug induced dyskinesias to be confused with MM. Only one patient who had been on a low dose of levodopa (200 mg/day) was included because confounding dyskinesias were not felt to be a concern.

\section{Clinical evaluation}

The initial assessment included ascertainment of handedness, duration of symptoms, side of first symptoms, and nature of earliest deficit. Patients underwent motor evaluation according to the motor subscale (part III) of the Unified Parkinson's Disease Rating Scale (UPDRS). ${ }^{16}$ Four tasks performed using the most affected limbs were videotaped: tapping of thumb against index finger (finger tapping, UPDRS item 23), rapid hand opening and closing (hand

Abbreviations: LAA, least affected arm; LAL, least affected leg; MAA, most affected arm; MAL, most affected leg; MM, mirror movements; PD, Parkinson's disease; UPDRS, Unified Parkinson's Disease Rating Scale 
Table 1 Scale used to assess severity of mirror movements in the resting less affected or unaffected hand and foot

\begin{tabular}{|c|c|c|}
\hline Features & Characteristics of mirroring & Score \\
\hline \multirow[t]{5}{*}{ Amplitude of mirroring } & No movement & 0 \\
\hline & Barely discernible but repetitive movement & 1 \\
\hline & $\begin{array}{l}\text { Clear movement with excursion of finger, wrist or ankle of less than } 2 \mathrm{~cm} \text {. Movement is either slight } \\
\text { but sustained or stronger but briefer }\end{array}$ & 2 \\
\hline & $\begin{array}{l}\text { Excursion of finger, wrist, or ankle greater than } 2 \mathrm{~cm} \text { and movement readily distinguished as } \\
\text { mirroring (strong and sustained movement) }\end{array}$ & 3 \\
\hline & Pronounced mirroring, with amplitude approaching or matching the requested task in the opposite limb & 4 \\
\hline \multirow[t]{3}{*}{ Distribution of mirroring } & Less than mirrored task (fewer fingers for instance) & 1 \\
\hline & Mirroring that involves the same areas as intended task & 2 \\
\hline & Mirroring spreads beyond the intended task areas & 3 \\
\hline \multirow[t]{4}{*}{ Proportion of mirroring } & Mirroring occurs in less than $1 / 3$ of the task intended cycles & 1 \\
\hline & Mirroring occurs in between $1 / 3$ and $2 / 3$ of the task cycles & 2 \\
\hline & Mirroring occurs for most of the task cycles ( $>2 / 3$ of the task cycles) & 3 \\
\hline & Maximum possible scoring: 40 (10 points/task) & \\
\hline
\end{tabular}

movements, UPDRS item 24), rapid hand pronation-supination movements (rapid alternating movement of hands, UPDRS item 25), and rapid ankle flexion-extension (foot tapping, UPDRS item 26). The videotape protocol required patients to sit on an examination table and perform each task for 15 cycles using their more affected limb at a rate of approximately $1 \mathrm{~Hz}$. The resting, less affected or unaffected forearm was supported in the lap with the hand held in neutral position, approximately halfway between pronation and supination. The ipsilateral foot dangled unsupported. These arm and leg positions were adopted because pilot testing suggested that mirroring was less overt when subjects had their limbs outstretched or resting on a bedside tray above the level of their waist. Subjects were unaware that our observations were focused on the resting hand and foot because of the concern that such information may have altered the MM phenomenology. The video camera was positioned sufficiently far away to include both arms for assessment of hand $\mathrm{MM}$ and subsequently both legs to evaluate foot MM, preventing patients from becoming aware of our attention on the non-active limb.

\section{Assessment of parkinsonism and mirroring phenomena}

To evaluate the regional components of parkinsonism, we used selected items of the UPDRS to determine axial and lateralised deficits. The axial UPDRS subscore was derived from the sum of subjective items $5,7,12,13,14$, and 15 from part II and motor exam items 18, 19, 22 (neck only), 27, 28, 29, and 30 from part III. The lateralised UPDRS subscore was evaluated in two ways: (1) the absolute difference between the motor deficits derived from the sum of items 20-26 (22, limbs only) restricted to one side and the sum of those items from the opposite side (for example, $(L-R)$ ); and (2) the ratio of this absolute difference and the sum of the scores from the two sides (for example, $(L-R) /(L+R)$ ). A larger

Table 2 Characteristics of enrolled patients

\begin{tabular}{ll}
\hline Characteristic & Mean (SD; range) \\
\hline Age at enrolment & $59.0(9.9 ; 39-82)$ \\
Age at disease onset & $56.7(9.6 ; 36-78)$ \\
Disease duration (month) & $27.8(14.7 ; 6-66)$ \\
Motor UPDRS & $18.9(7.7 ; 10-36.5)$ \\
Axial UPDRS & $5.8(3.3 ; 0-14.95)$ \\
Lateralised UPDRS & $7.7(3.7 ; 1-14)$ \\
\hline \multirow{2}{*}{ UPDRS, Unified Parkinson's Disease Rating Scale. }
\end{tabular}

lateralised UPDRS score indicates greater difference of disease burden between sides and, therefore, more asymmetry. In addition, we calculated a composite rigidity-bradykinesia score for each limb. For the upper extremities, the scores for the more affected (MAA) and less affected (LAA) arm were obtained as follows:

MAA $=($ UPDRS item 22$)+(($ UPDRS items $23+24+25) / 3)$ for more affected arm.

$\mathrm{LAA}=($ UPDRS item 22$)+(($ UPDRS items $23+24+25) / 3)$ for less affected arm.

Similarly, for the lower extremities, the scores for the more affected (MAL) and less affected (LAL) leg were calculated as follows:

MAL $=($ UPDRS item $22+$ UPDRS item 26) for more affected leg.

$\mathrm{LAL}=($ UPDRS item $22+$ UPDRS item 26) for less affected leg.

An investigator, unaware of the clinical variables collected separately, determined the severity of the mirroring. This entailed blinded videotape assessment using a scale that rated amplitude, distribution, and proportion of mirroring activity in the less affected limbs (table 1; for representative mirroring subjects see video available at http:// www.jnnp.com/supplemental).

The three item scale included a measure of amplitude (defined as the range of excursion of the fingers and wrist (for hand MM) and ankle (for foot MM)), distribution (the extent to which these movements matched those of the joints and muscles of the task performing limb), and proportion (the approximate fraction of time during which the mirroring occurred during task performance). When in doubt about the degree of mirroring, the lower most appropriate rate for the movement was used. Because there were three tasks in the potentially mirroring hand (UPDRS items 23-25) and one in the ipsilateral foot (UPDRS item 26), the total possible score was 40. Our cohort exhibited a range of $0-31$.

Table 3 Clinical aspects of enrolled patients

\begin{tabular}{ll}
\hline Clinical aspects & Patient number (percentage of total) \\
\hline Side of first symptoms & $\begin{array}{l}\text { Left, 13 }(48 \%) ; \text { right, 13 (48\%); bilateral, 1 } \\
(4 \%)\end{array}$ \\
Nature of first symptom & $\begin{array}{l}\text { Tremor, 19 }(70 \%) ; \text { bradykinesia, } 5(19 \%) ; \\
\text { micrographia, 2 (7\%) }\end{array}$ \\
Drug treatment & $\begin{array}{l}\text { Pramipexole, 2 (7\%); ropinirole, 2 }(7 \%) ; \\
\text { levodopa, 1 }(4 \%) ; \text { no treatment, } 22(81 \%)\end{array}$ \\
\hline
\end{tabular}




\section{Data analysis}

Simple and multiple linear regressions were used to test the effects of UPDRS total motor, axial, limb composites, and lateralised scores on MM. Pearson correlation coefficients were obtained between the mirroring score and the lateralised UPDRS. Analysis of covariance (ANCOVA) was applied to determine the predictive effect of age, sex, nature of initial symptom, side of initial symptom, dopaminergic treatment, and duration of disease on the degree of mirroring. The Mann-Whitney U test was used to determine differences of baseline parkinsonian deficits based on sex and nature and side of initial symptom. SAS statistical software version 8.2 and StatView version 5.01 (SAS Institute Inc, Cary, North Carolina, USA) were used for analyses and graphics.

\section{RESULTS}

\section{Baseline characteristics}

Tables 2 and 3 list the demographic features of the 27 patients. The side of the first symptom, the nature of their first symptom, age, and sex had no influence on MM score, disease duration, or UPDRS score.

\section{General features of mirroring}

MM were easily discernible in the less affected limb in 24 of the 27 early asymmetric patients with PD. No MM were noted in the most affected limb while the patient performed activities in the less affected limb. Mirroring was present only in the hand in 10 of 24 and in the foot in two of 24 . Half of our mirroring subjects (12 of 24) had both hand and foot MM. Of these, mirroring was more predominant in the less affected hand in seven of 12 and in the less affected foot in five of 12 . Hand mirroring was slightly more common during finger tapping (mean score, 5.23; maximum possible, 10) than during hand movements (mean, 4.04) or forearm pronation-supination (mean, 4.91). The mean (SD) total MM score was 14.8 (9.3) and was higher in those whose presenting deficit was tremor (mean, 15.5; SD, 10.1) than bradykinesia (mean, 8.3; SD, 3.7). Mirroring in the resting limb could be conspicuous during one task (for example, finger tapping) but absent during another (for example, pronation-supination of the forearm).

\section{Relation between mirroring and parkinsonian features}

The lateralised UPDRS score predicted the severity of mirroring in the less affected or unaffected side in a linear fashion $(R=0.56 ; \mathrm{p}=0.005 ;$ fig $1 \mathrm{~A})$, and this finding remained significant even when the three subjects without mirroring were included $(R=0.39 ; \mathrm{p}=0.046)$. When using an index of asymmetry by determining the ratio between absolute differences of motor deficits between sides and the total motor impairment, a significant direct correlation between this index and the MM scores was found $(R=0.728 ; \mathrm{p}<0.001$; fig $1 \mathrm{~B})$, indicating that the higher the absolute difference between sides in relation to the total impairment (higher ratio), the more prominent the mirroring observed. Conversely, patients with a greater burden of motor impairment and more symmetry of bilateral deficits (lower ratio) exhibited little or no mirroring. In other words, given two individuals with similar absolute motor differences between sides, greater mirroring was present in the individual who exhibited a greater proportional difference, as is the case at the milder end of the motor impairment spectrum (for example, approaching the state of pure hemiparkinsonism). Although there was a trend for an inverse correlation between the total UPDRS and MM, no correlation was found between MM and the modified axial UPDRS or the duration of disease.

To determine regional predictors of mirroring, we first evaluated the influence of the combined rigidity-bradykinesia
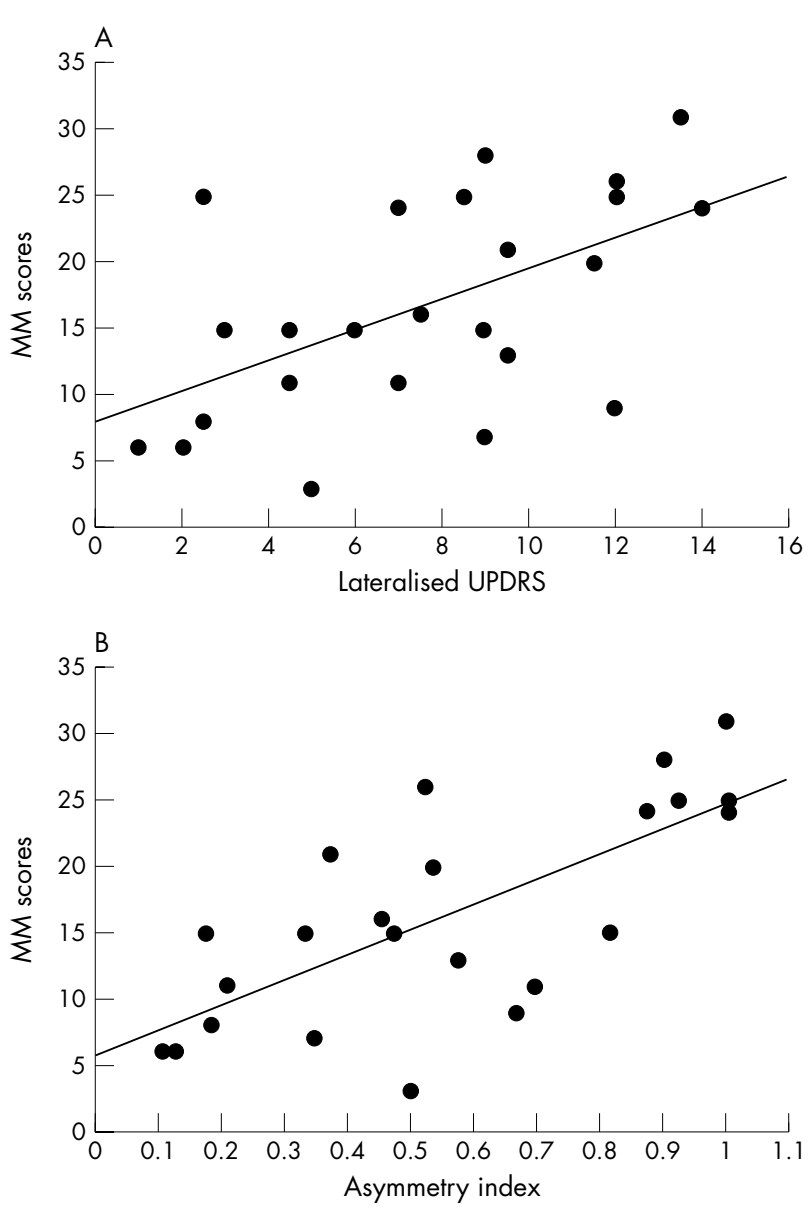

Figure 1 (A) Regression analysis between mirror movement (MM) scores and lateralised Unified Parkinson's Disease Rating Scale (UPDRS). Lateralised UPDRS is the absolute difference of motor deficits between sides. Three patients without MM were removed from this analysis. (B) Regression analysis between $M M$ scores and the asymmetry index. The asymmetry index was defined as the ratio between absolute differences of motor deficits between sides divided by the total motor impairment, according to the following formula: lateralised UPDRS ratio = $($ UPDRS left - UPDRS right)/(UPDRS left + UPDRS right)). Three patients without $M M$ were removed from this analysis.

composite on MAA, MAL, LAA, and LAL scores. Foot mirroring, but not hand mirroring, was predicted when both leg composites were considered together in a multiple regression analysis $(R=0.55$; MAL, $\beta=3.60, \mathrm{p}=0.02$; and LAL, $\beta=-5.92, p=0.004)$. This suggested that the more severe the core motor deficits (rigidity and bradykinesia) in the more affected leg, the greater the correlation with foot mirroring in the less affected leg, particularly if this limb exhibited minimal motor deficits.

When differences in bradykinesia and rigidity between sides were considered in separate linear regression models, a significant direct correlation was found only between lateralised rigidity in the legs and foot $\mathrm{MM}(R=0.66$; $\mathrm{p}<0.001)$. Differences in rigidity or bradykinesia between arms failed to predict hand mirroring.

\section{DISCUSSION}

We have found that MM are a feature of asymmetric parkinsonism, particularly when overall motor impairment is relatively minor. Hand and foot mirroring were predicted by the difference in motor deficits between sides. Differential rigidity in the legs was significantly associated with foot mirroring. Because no relation was found between MM and the total motor UPDRS score, a modified axial score, or the duration of disease, the 
phenomenon is not necessarily a feature of early disease, but instead predominantly a feature of asymmetric disease, in which the more affected side has higher motor scores, whereas the least affected side is minimally or not involved at all.

One report has been published regarding the association of mirror movements and hemiparkinsonism. ${ }^{15}$ This was based on the elicitation of mirroring during UPDRS bradykinesia tasks, and was similar to our original observation ${ }^{14}$ that MM occur in the relaxed less affected or unaffected hand while voluntary movements are performed with the more affected hand. In addition, their study in 11 untreated patients with hemiparkinsonism found a significant correlation between the UPDRS motor score and the "occurrence" of MM using a dichotomous outcome of its presence or absence. Contrary to this, we had reported a tendency for an inverse correlation between total motor UPDRS and the severity of mirroring. ${ }^{14}$ Our larger sample reported here is consistent with this trend, although the negative coefficient did not reach significance. Nevertheless, our data suggest instead that it is the differential severity of motor impairment between sides, as demonstrated by the strong effect of the absolute and relative UPDRS lateralised scores, that best predicts mirroring on the less affected side. Because Vidal et al studied patients with pure hemiparkinsonism with a mean motor UPDRS of 12 (compared with 19 in our cohort), their UPDRS scores are practically equivalent to the lateralised measure we defined for our evaluation. Had patients with more parkinsoniam impairment of the "unaffected" side been included in their analysis, the non-lateralised motor UPDRS might have failed to predict mirroring.

MM of hands and fingers are normally seen in early childhood and fade with age. ${ }^{17}$ Their persistence has been associated with various disorders that presumably impair interhemispheric pathways involved in limb motor control. In the most studied disorder associated with MM, X linked Kallmann's syndrome, ${ }^{18}$ the agenesis of the corpus callosum and, possibly, the absence of pyramidal decussation support this hypothesis. Among acquired disorders, stroke has received considerable attention. ${ }^{19-21} \mathrm{MM}$ occur most often in the unaffected hand, especially among those with greater motor deficits but also, to a lesser extent, in the paretic hand. Interestingly, $\mathrm{MM}$ in the paretic hand have been associated with better motor function. ${ }^{19}$ Increased activation of the unaffected motor cortex with preserved activation in the stroke hemisphere has been correlated with MM in the unaffected hand. ${ }^{22}$ This is supported by transcranial magnetic stimulation and $\mathrm{H}_{2}{ }^{15} \mathrm{O}$ positron emission tomography activation studies of primary MM syndromes, where enhanced facilitation and increased cerebral blood flow of the ipsilateral motor cortex has been found. ${ }^{21}$ Therefore, MM may arise from increased activity of the ipsilateral cortical hand projections or, alternatively, from reduced transcallosal inhibition or increased transcallosal facilitation. At present, the underlying pathophysiology of MM in parkinsonism remains undetermined, but is almost certainly distinct from that resulting in the persistent $\mathrm{MM}$ related to pyramidal abnormalities present in congenital mirror movements, Klippel-Feil syndrome, X linked Kallmann's syndrome, or other structural abnormalities.

Given the restrictions of our study design, we cannot comment on how often these movements are present in patients with early parkinsonism. Based on our clinical observations, however, we have been impressed that when carefully sought, they are evident in most patients with asymmetrical clinical features. We have also found that their absence is a useful feature in patients with very asymmetrical or unilateral psychogenic parkinsonism. The natural evolution of MM is uncertain. Our results would predict that the increasing symmetry of disease with longer disease duration should progressively decrease their presence. A longitudinal study will be needed to establish the effect of disease progression and dopaminergic treatment on the mirroring phenomenon. However, in later stage parkinsonism, levodopa induced dyskinesias may impair the assessment of the presence of MM, especially because dyskinesias tend to occur earlier in the most affected side. Here, "overflow" dyskinesias induced by activation of the other limb may confound the mirroring phenomenology. We are also unable to comment on the prognostic relevance that MM might have. All patients in our study had a diagnosis of PD and some were subsequently confirmed to have an adequate response to levodopa or dopamine agonists. However, sufficiently longterm follow up would have been required to exclude other causes of parkinsonism. It is our impression that MM correlate with clinical asymmetry and not with diagnosis because we have also seen MM in patients with asymmetric parkinsonism caused by multiple system atrophy.

In summary, MM of the unaffected or less affected hand and foot are a feature of relatively mild asymmetrical parkinsonism, especially dominated by rigidity. Further studies may shed light on the potential value of MM in categorising and staging patients with early parkinsonism.

\section{Authors' affiliations}

A J Espay, J-Y Li, L Johnston, R Chen, A E Lang, Division of Neurology, Department of Medicine, Toronto Western Research Institute, University of Toronto, Toronto, Ontario MST 258, Canada

Competing interests: none declared

\section{REFERENCES}

1 Schott GD, Wyke MA. Congenital mirror movements. J Neurol Neurosurg Psychiatry 1981;44:586-99.

2 Radhakrishnan K, Koshy E, Prakash C. Ataxic hemiparesis and mirror movements. J Neurol Neurosurg Psychiatry 1981;44:190.

3 Gardner WJ. Klippel-Feil syndrome, iniencephalus, anencephalus, hindbrain hernia and mirror movements: overdistention of the neural tube. Childs Brain 1979;5:361-79.

4 Farmer SF, Ingram DA, Stephens JA. Mirror movements studied in a patient with Klippel-Feil syndrome. J Physiol 1990;428:467-84.

5 Glos J, Bzduch V, Lisy L, et al. Large mirror movements of upper extremities in Williams syndrome. Pediatr Neurol 1993;9:414.

6 Krams M, Quinton R, Ashburner J, et al. Kallmann's syndrome: mirror movements associated with bilateral corticospinal tract hypertrophy. Neurology 1999;52:816-22.

7 Odabasi Z, Gokcil Z, Kutukcu Y, et al. Mirror movements associated with cervical meningocele: case report. Minim Invasive Neurosurg 1998;41:99-100.

8 Erdincler $\mathrm{P}$. Cervical cord tethering and congenital mirror movements: is it an association rather than a coincidence? Br J Neurosurg 2002;16:519-22.

9 Geffen GM, Jones DL, Geffen LB. Interhemispheric control of manual motor activity. Behav Brain Res 1994;64:131-40.

10 Aranyi Z, Rosler KM. Effort-induced mirror movements. A study of transcallosal inhibition in humans. Exp Brain Res 2002;145:76-82.

11 Pohja M, Salenius S, Hari R. Cortico-muscular coupling in a human subject with mirror movements - a magnetoencephalographic study. Neurosci Lett 2002;327:185-8

12 Netz J, Lammers T, Homberg V. Reorganization of motor output in the nonaffected hemisphere after stroke. Brain 1997;120:1579-86.

13 Fisher CM. Alien hand phenomena: a review with the addition of six personal cases. Can J Neurol Sci 2000;27:192-203.

14 Espay AJ, Li J, Chen R, et al. Subtle mirror movements in hemiparkinsonism: a useful clinical sign (preliminary observations). Mov Disord 2002; 17:S261.

15 Vidal JS, Derkinderen P, Vidailhet $M$, et al. Mirror movements of the nonaffected hand in hemiparkinsonian patients: a reflection of ipsilateral motor overactivity? I Neurol Neurosurg Psychiatry 2003;74:1352-3.

16 Fahn S, Elton RL. Unified Parkinson's Disease Rating Scale. In: Fahn S, Goldstein M, Marsden D, et al. Recent developments in Parkinson's disease. New Jersey: MacMillan, 1987:153-63.

17 Largo RH, Caflisch JA, Hug F, et al. Neuromotor development from 5 to 18 years. Part 2: associated movements. Dev Med Child Neurol $2001 ; 43: 444-53$.

18 Krams M, Quinton R, Ashburner J, et al. Kallmann's syndrome: mirror movements associated with bilateral corticospinal tract hypertrophy. Neurology 1999;52:816-22.

19 Nelles G, Cramer SC, Schaechter JD, et al. Quantitative assessment of mirror movements after stroke. Stroke 1998;29:1182-7.

20 Chaco J, Blank A. Mirror movements in hemiparesis. Confin Neurol 1974;36:1-4.

21 Kanouchi T, Yokota T, Isa F, et al. Role of the ipsilateral motor cortex in mirror movements. J Neurol Neurosurg Psychiatry 1997;62:629-32.

$22 \mathrm{Kim}$ YH, Jang SH, Chang Y, et al. Bilateral primary sensori-motor cortex activation of post-stroke mirror movements: an fMRI study. Neuroreport 2003;14:1329-32. 
Feeding cats might be dangerous: penetrating orbital and brain injury without neurological deficits

A 67 year old alcoholised woman stumbled over an unevenness, lost her balance, and fell into an ordinary butterknife while preparing cat food.

Initially the woman was awake with a Glasgow coma scale (GCS) of 9. On hospital admission, she was intubated, sedated, the pupils were equal and reactive to light, the eyeballs were both intact, and there was no neurological deficit. The handle of the knife was protruding from the right orbit. There was a leakage of bloody cerebrospinal fluid (CSF) out of the inner right canthus.

$\mathrm{X}$ ray and computed tomography $(\mathrm{CT})$ demonstrated the blade penetrating the medial part of the right orbit-gyrus rectus extending to the anterior horn of the right lateral ventricle (fig 1). Beside there was an old infarction area due to the right medial cerebral artery. After exclusion of a space occupying intracerebral haemorrhage the knife was pulled out in the operating theatre. After removing the knife a control CT was performed revealing only a minimal traumatic subarachnoid haemorrhage ( $\mathrm{tSAH}$ ) (fig 2). Thereafter, the right canthus was microsurgically sutured.

Postoperative contrast-enhanced, T1 and T2 weighted magnetic resonance images revealed minimal tSAH and a slight defect zone in the right gyrus rectus (fig 3). Hypothalamus, pituitary gland, and optic nerve were completely unaffected. Initially, CSF analysis indicated massive erythrocytes (due to the tSAH), a disturbed bloodbrain-barrier function, and a total cell count of $38 / \mathrm{mm}^{3}$. Hormonal testing was inconspicious including prolactin, follicle-stimulating hormone, lutheinizing hormone, growth hormone, thyrotropin, and corticotropin. Electrolytes were normal and the patient did not develop a syndrome of inappropriate secretion of antidiuretic hormone (SIADH). Neuroophtalmologic investigation revealed no loss of visual acuity and full ocular motility.

She received a prophylactc antibiotic therapy including amoxicillin/clavulanate $(3 \times 2.2 \mathrm{~g})$, ciprofloxacin $(2 \times 0.5 \mathrm{~g})$, and metronidazole $(2 \times 0.4 \mathrm{~g})$. Through the clinical course the

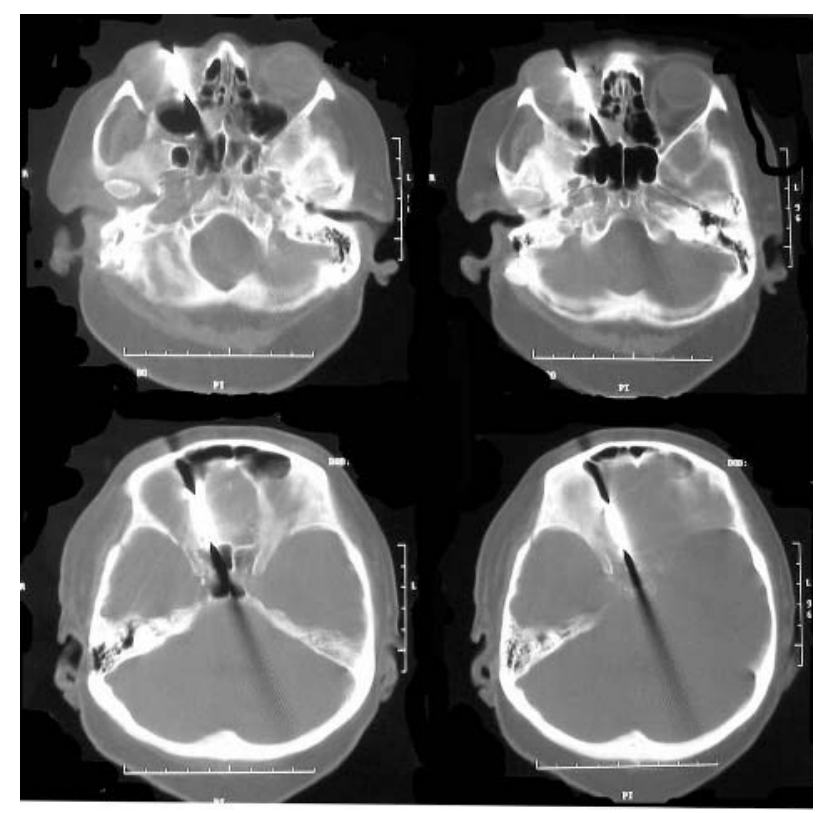

Figure $1 \mathrm{CT}$ with bone algorithm: butterknife penetrating through the orbit, frontal cranial base, and frontal lobe.

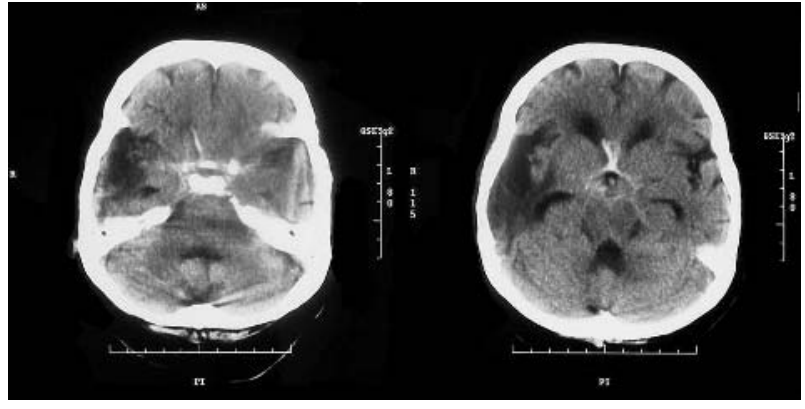

Figure 2 Postoperative native $\mathrm{CT}$ demonstrating $\mathrm{tSAH}$. Note the defect zone resulting from an ischaemic infarct.
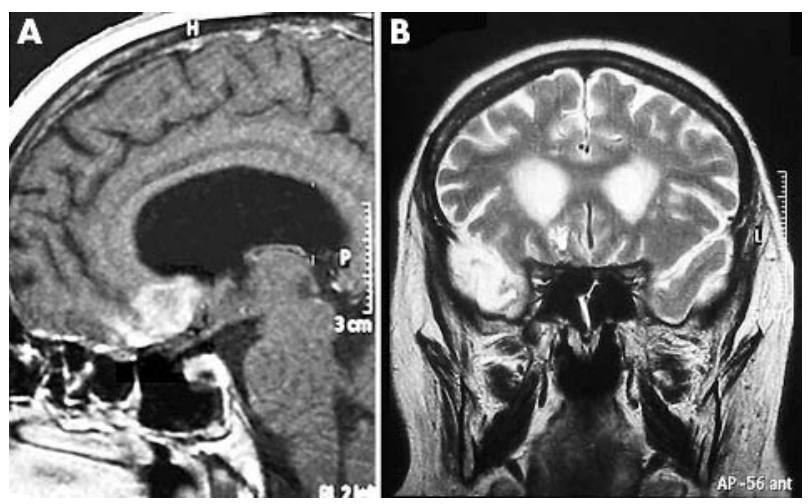

Figure 3 Postoperative magnetic resonance images (left $\mathrm{Tl}$ sagittal, right $\mathrm{T} 2$ coronal): beside $\mathrm{H} \mathrm{SAH}$ there was a slight defect zone in the right gyrus rectus. No lesions became obvious in the hypothalamus, pituitary gland, and optic nerve.

patient showed no signs of infection. As an independent complication she developed a crural thrombosis and was discharged on day 37 after initial trauma.

Penetrating orbital trauma should alert the clinician because of potentially devastating consequences. Even an intact orbital globe does not exclude intracranial trauma. The high mortality rate associated with cranial penetration injuries is mainly a result of intracranial suppuration. ${ }^{1-3}$ Therefore, an appropriate, broad spectrum antimicrobial therapy is inevitable. Considering the serious injury the patient's course was remarkably inconspicuous.

\section{F Knerlich, R Verheggen University Hospital of Göttingen, Göttingen, Germany}

Correspondence to: R Verheggen, Department of Neurosurgery, University hospital, Georg-August-University of Göttingen Göttingen, 37075, Germany; raphaela.verheggen@med.uni-goettingen.de

Competing interests: none declared

\section{References}

1 Duffy GP, Bhandari YS. Intracranial complications following transorbital penetrating injuries. Br J Surg 1969;56:685-8.

2 Verin P, Vildy A, Benjelloun D. Menigitis caused by orbital injuries. Bull Soc Ophthal Fr 1976;76:969-70.

3 Wesley RE, Anderson SR, Weiss MR, et al. Management of orbital-cranial trauma. Adv Ophthal Plast Reconstruct Surg 1988;7:3-26. 